\title{
La nutraceutiques (alicaments) et compléments alimentaires dans le traitement de l'infertilité masculine
}

\author{
Frank COMHAIRE, Ahmed MAHMOUD \\ Centre d'andrologie et d'endocrinologie reproductive, Gand. Belgique
}

\begin{abstract}
RESUME
Plusieurs essais contrôlés et correctement validés apportent des preuves que des compléments alimentaires contenant des substances particulières peuvent améliorer la qualité et la fonction spermatiques d'hommes hypofertiles. Parmi ces compléments figurent les antioxydants astaxanthine, tocophérols et ubiquinone Q10, des acides gras essentiels du groupe oméga 3, le zinc et l'acide folique. II y a des études qui suggèrent que certains extraits de plantes et des lignans ont également des effets bénéfiques. L'administration d'un nutraceutique (Qualisperm B, Nutriphyt, Oostkamp, Belgique) contenant ces substances augmente la probabilité d'une conception spontanée et d'une grossesse menée à terme. Bien que les mécanismes exacts de l'action de ces compléments alimentaires sur la spermatogenèse et la fonction spermatique n'aient pas encore été entièrement découverts, il semble concevable que ces compléments agissent directement sur les cellules de Sertoli et améliore la fonction épididymaire. En tant que compléments alimentaires exempts de toute toxicité, les nutraceutiques devraient également être pris en considération avant de procéder à une fertilisation in vitro et à une ICSI, afin de réduire les dégâts oxydatifs à I'ADN des spermatozoïdes.
\end{abstract}

Mots clés : nutraceutiques, alicaments, infertilité, antioxydants, ADN oxydé, acides gras

\section{INTRODUCTION}

Le lancement de la technologie reproductive assistée (TRA), notamment la fécondation in vitro (FIV) [65] et l'injection intracytoplasmique de spermatozoïdes (ICSI) [51] ont été à l'origine d'une véritable révolution dans la médecine reproductive tout en révélant également l'ampleur de la contribution du "facteur masculin" à l'infertilité du couple. Le traitement classique de l'infertilité masculine a été considéré comme dépassé par certains, mais d'autres ont continué à débrouiller les mécanismes de la capacité reproductrice défectueuse de I'homme. Ces dernières années, des objections ont été formulées concernant les aspects économiques et éthiques $[10,33]$ et les effets indésirables de la technologie TRA $[38,59]$. On a observé que la FIV et I'ICSI sont associées à une prévalence accrue de défauts génétiques [20], à des malformations congénitales majeures $[26,27,34,37,67,71,78]$, à un développement perturbé $[52,66]$, et à un risque accru de rétinoblastomes [49] ainsi que d'autres tumeurs malignes $[18,43]$ chez la progéniture. Aujourd'hui, la boucle semble bouclée et l'andrologie clinique semble avoir retrouvé une place bien méritée au sein de la panoplie d'instruments permettant de traiter l'infertilité du couple.

\section{L'INFLUENCE DU MODE DE VIE ET DES FACTEURS NUTRITIONNELS}

Au même titre que d'autres maladies, l'infertilité masculine est une maladie dont l'évolution résulte d'une série de facteurs pathogéniques. Ici quatre principaux groupes de facteurs semblent agir en synergie: défauts génétiques ou constitutionnels, facteurs liés au mode de vie, exposition à des risques professionnels et/ou environnementaux, maladies de l'appareil uro-génital ou du système endocrinien.

Le domaine de la génétique se développe rapidement et comporte des anormalités numériques et structurales des chromosomes ainsi que des micro-délétions du chromosome $Y[42,69]$. Que certaines micro-délétions génétiques puissent se manifester sous forme d'infertilité peut dépendre de la présence coïncidente de facteurs de mode de vie défavorables ou de l'exposition à des substances toxiques ainsi qu'à des pertubateurs hormonaux. On a

\section{Correspondance :}

Dr Frank COMHAIRE - Centre d'andrologie et d'endocrinologie reproductive, Hôpital Universitaire de Gand, De Pintelaan 185, B-9000 Gand, Belgique - Tel +32 92402133 Fax +3292403897 - Email frank.comhaire@ugent.be 
démontré que ces derniers, ainsi que les maladies génitales, accroissent la surcharge d'espèces d'oxygène réactif (reactive oxygen species - ROS) dans l'éjaculat et les spermatozoïdes, résultant en un fractionnement accru des chromosomes $[28,30]$ et une production excessive d'ADN oxydé (8-hydroxy 2-deoxy guanosine) [21]. Cette dernière induit une mutagenèse de transition.

La membrane des spermatozoïdes de l'homme fertile contient une concentration élevée en acide docosahexaenoïque (DHA, appelé également : acide cervonique, 22:6w3) qui confère sa fluidité à la membrane. La fluidité est indispensable pour permettre la réaction acrosomique et la fusion des membranes de la tête du spermatozoïde et de l'ovocyte. La membrane du spermatozoïde de l'homme infertile contient moins de DHA, réduisant ainsi la fluidité et la capacité fusiogénique. Cependant, l'acide DHA présente une grande susceptibilité oxydative en raison du nombre élevé de doubles liaisons. D'aure part, le sérum de l'homme infertile présente un déséquilibre entre la surcharge oxydative et une moindre capacité antioxydative, qui a pu être démontré par la sensibilité oxydative accrue du cholestérol LDL chez l'homme infertile par rapport à l'homme fertile. La surcharge oxydative modifie la composition phospholipidique de la membrane du spermatozoïde [81], réduisant sa teneur en acide DHA et sa fluidité, ce qui se traduit par une diminution tant de la capacité fusiogénique que de la réactivité acrosomique.

Une alimentation inappropriée, l'abus d'alcool, de tabac ou de drogues récréatives, des vêtements trop serrés et les bains chauds figurent parmi les facteurs de mode de vie incriminés. En outre, on a observé qu'une proportion plus élevée d'hommes ayant une qualité de sperme déficiente présentent un indice de masse corporelle dépassant la valeur optimale de 25 et sont en surcharge pondérale ou franchement obèses. Ils consomment moins d'acides gras oméga 3 que les hommes fertiles, et le quotient des acides oméga 3 divisé par les acides oméga 6 est généralement inférieur au quotient optimal. Une corrélation positive significative a été établie entre la consommation d'acide alpha linolénique (18:3 w3) d'un côté, et la concentration de spermatozoïdes et la motilité de type (a) de l'autre côté [8]. Par contraste, il y avait une corrélation négative entre ces caractéristiques spermatiques et la consommation d'acides gras hautement poly-insaturés ( $y$ compris l'acide DHA). Ceci suggère que la surcharge oxydative, présente chez I'homme infertile, peut induire la cascade oxydative en cas de consommation élevée d'acides gras poly-insaturés, plus vulnérables que les acides gras à chaîne courte et moins insaturés, tels que l'acide alpha linolénique. Enfin, il a été démontré que le tissu testiculaire, en particulier les cellules de Sertoli, présente une teneur plus élevée en désaturase [58] et élongase [9] que d'autres tissus organiques; ces enzymes transforment l'acide alpha linolénique en acides gras polyinsaturés à chaîne longue $[29,70]$. Ce processus génère des concentrations de DHA locales plus élevées.

Il a été démontré que la qualité des spermatozoïdes est affectée par l'exposition à des substances toxiques dans un environnement professionnel, notamment à des métaux lourds tels que le plomb [6], et le disulfure de carbone [73]. Mais c'est surtout l'exposition à des agents environnementaux entraînant un dérèglement hormonal, principalement aux pseudo ou xéno-œstrogènes et aux anti-androgènes, qui a suscité beaucoup d'inquiétude ces derniers temps. La détérioration manifeste, bien que régionale, tant des variables spermatiques que de la fertilité, et l'augmentation parallèle de la prévalence du cancer des testicules, ont été mis en rapport avec une exposition interne accrue à des substances chimiques, fabriquées par l'homme, qui reproduisent ou amplifient les effets des œstrogènes en se fixant sur le récepteur d'œstrogène humain ou en influençant le métabolisme des œstrogènes [pour une revue voir : $62,63]$.

\section{LA FONCTION PIVOT DE L'INHIBINE B}

L'Inhibine $B$ est un produit de sécrétion des cellules de Sertoli qui joue un rôle important tant dans la rétro-régulation endocrinienne, inhibant la sécrétion hypophysaire de $\mathrm{FSH}$, que dans la régulation locale de la spermatogenèse. $\mathrm{Si}$, normalement, la concentration d'Inhibine B dans le sérum est significativement liée à la concentration des spermatozoïdes [pour une revue voir : 47], il y a des preuves d'un effet de suppression directe par l'Inhibine B sur la spermatogenèse [72]. Les tests in vitro [19] comme les données in vivo $[44,45]$ indiquent que les œstrogènes et certains métaux lourds, tel le plomb, pourraient stimuler de façon inappropriée la sécrétion d'Inhibine B par les cellules de Sertoli. Ceci entraîne une production de spermatozoïdes abaissée, en présence de concentrations normales d'Inhibine B et de FSH dans le sérum.

Pendant le traitement avec le puissant antioxydant Astaxanthine, qui réduit la concentration des radicaux oxygène libres (ROS) dans l'éjaculat, la concentration d'Inhibine $B$ dans le sérum était abaissée, bien que la concentration du sperme soit restée inchangée [13]. Ceci suggère que les ROS stimulent la sécrétion d'Inhibine B par les cellules de Sertoli, d'une manière comparable à l'effet des œstrogènes.

L'abaissement de la sécrétion d'Inhibine $B$ par une réduction de la surcharge d'œstrogène et l'exposition aux ROS pourrait donc être un objectif de traitement médical.

\section{COMPLEMENTS ALIMENTAIRES}

\section{Acides gras}

Étant donné qu'il y a une corrélation positive entre la prise nutritionnelle d'acide alpha linolénique (ALA) et la concentration et la motilité des spermatozoïdes, et puisque chez les hommes à fertilité diminuée, la prise d'acides gras essentiels du groupe oméga 3 s'est avérée inférieure à la prise optimale, il semble logique d'administrer à ces patients des suppléments d'acide ALA, notamment en leur donnant de l'huile de graines de lin. Lorsqu'il est administré en combinaison avec les cofacteurs Zinc et vitamine B6, qui stimulent l'activité des enzymes élongase et désaturase, l'acide alpha linolénique se transforme in situ en acides 
gras oméga 3 hautement insaturés à chaîne longue, notamment en acide eicosapantaénoïque (EPA) et docosahexaénoïque (DHA). La fluidité de la membrane des spermatozoïdes sera améliorée et la réaction acrosomique et la capacité fusiogénique des spermatozoïdes augmenteront [12].

Alternativement, l'administration d'huile de poisson peut être considérée comme une source d'acides gras hautement insaturés à chaîne longue EPA et DHA. Cependant, ces acides gras sont extrêmement sensibles aux dommages oxydatifs entraînant une réaction en chaîne de lipooxydation indésirable. Si de l'huile de poisson est administrée comme complément alimentaire, il est impératif d'assurer un environnement interne antioxydant favorable.

\section{Antioxydants}

On a observé qu'il y a chez les patients hypofertiles un déséquilibre entre un stress oxydatif excessif par comparaison à une capacité antioxydante réduite [8]. L'administration de compléments alimentaires contenant des antioxydants améliore significativement et durablement l'équilibre entre une surcharge oxydative et la défense antioxydante [5]. Le traitement, soit avec de l'acétylcystéine (600 mg par jour oralement) ou avec un mélange antioxydant, réduit significativement la teneur en radicaux d'oxygène réactif (ROS) dans le sperme [12]. Administré en combinaison avec de l'huile de poisson, le traitement antioxydant élève la concentration des spermatozoïdes et réduit significativement la concentration d'ADN oxydé $(8-\mathrm{OH}-2 \mathrm{~d}-\mathrm{Gua}$ nosine) dans les spermatozoïdes de l'homme hypofertile. La composition des acides gras dans les phospholipides de la membrane des spermatozoïdes évolue vers les acides EPA et DHA, ce qui augmente la fluidité de la membrane et se traduit par une réactivité acrosomique accrue induite, mais non spontanée. Ce traitement augmente le taux de conception spontané, en particulier chez les couples où l'homme hypo-fertile est fumeur. De plus, les suppléments de vitamine $E$ améliorent la fonction in vitro des spermatozoïdes comme en atteste le test avec des ovocytes de hamster défaits de leur zone pellucide (zona free hamster oocyte test) [35].

On a observé que l'administration de suppléments de vitamine $\mathrm{C}$ à des fumeurs présentant une qualité de sperme anormale améliore la qualité de leur sperme [16], mais cet effet n'a pas été observé lors d'un autre essai faisant usage d'une forte dose de vitamine $C$ [55]. Ceci peut être lié à l'effet pro-oxydatif connu des fortes doses de vitamine $C$ [21], en particulier chez des hommes dont l'haptoglobine est de type 1-2 ou 2-2 [5].

Si elle est ajoutée in vitro ou administrée oralement [41], l'oxydoréductase ubiquinone Q10 augmente la motilité des spermatozoïdes dans les cas d'asthénozoospermie. On a observé que d'autres antioxydants tels que le sélénium [61] et le glutathione [39] amélioraient également la motilité des spermatozoïdes chez des sous-groupes de patients.

L'Astaxanthine est un caroténoïde lipophile produit par l'algue Haematococcus pluvialis et possédant un grand pou- voir antioxydant [25, 31]. Lors d'un essai pilote randomisé en double aveugle, de l'Astaxanthine naturelle (AstaRealß, Gustavsberg, Suède) a été administrée aux partenaires masculins de couples infertiles présentant des caractéristiques spermatiques nettement inférieures aux valeurs de référence recommandées par l'O.M.S. L'administration de ce complément alimentaire a entraîné une réduction significative des ROS séminales et de la concentration d'inhibine $B$ dans le sérum chez des patients traités, alors qu'aucune modification ne s'est produite chez les individus témoins sous placebo. La motilité linéaire ou mouvement en avant rapide, a augmenté significativement et la morphologie des spermatozoïdes présentait une amélioration non significative dans le groupe de l'Astaxanthine. Dans le groupe des patients traités, les taux de grossesse total et mensuel étaient de respectivement $54,5 \%$ et $23,1 \%$ par rapport à $11,1 \%$ et $3,6 \%$ dans le groupe placebo [13].

\section{Carnitine}

La L-carnitine joue un rôle pivot dans les mécanismes de transport qui sont indispensables pour assurer le passage des acides gras à chaine longue entre le cytosole cellulaire et la matrice mitochondriale où ils sont oxydés, générant de l'énergie [79] et stimulant les complexes de la chaîne respiratoire de Krebs [57]. La carnitine libre et l'acétyl Lcarnitine jouent un rôle important dans la maturation postgonadique des spermatozoïdes des mammifères [32], et le quotient acétyle L-carnitine/carnitine était différent dans de spermatozoïdes de bonne ou de mauvaise qualité $[3,22]$. L'acétyle L-carnitine est la carnitine dominante dans les spermatozoïdes et sa concentration était inférieure dans le sperme d'hommes infertiles [36, 64]. La concentration de carnitine libre dans le plasma séminal était en corrélation significative avec la concentration et la motilité spermatiques [48], et il a été possible de stimuler la motilité des spermatozoïdes en ajoutant de l'acétyle carnitine in vitro [68].

On a observé que le traitement au moyen d'un complément alimentaire contenant une combinaison de $L$ carnitine (2 grammes par jour) et d'acétyle L carnitine (1 gramme

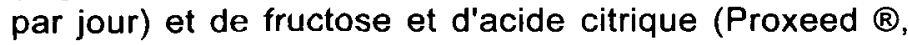
Sigma-tau Health Science, Rome, Italie), avait pour effet d'élever la concentration spermatique et la motilité progressive tant dans des études dites ouvertes [15, 50, 75] que dans une étude croisé en double aveugle [40]. Dans une des études ouvertes, on a enregistré un taux de grossesse de $6,7 \%$ en 3 mois [76]. Le taux de conception mensuel obtenu par méta-analyse d'essais publiés était de $2,3 \%$ [14]. Dans un essai en double aveugle, la prise complémentaire de Proxeed (B) n'a cependant pas influencé les résultats du traitement conventionnel, ni en termes de caractéristiques spermatiques ni en ce qui concerne le taux de grossesse (Comhaire et al., non publié).

\section{Acide folique et zinc}

De l'acide folique et du zinc ont été administrés oralement tant à des hommes présentant un sperme de qualité normale qu'à des patients atteints d'une oligozoospermie modérée durant un essaj contrôlé contre placebo [80]. On 
a observé que cette combinaison augmente significativement la concentration en spermatozoïdes (d'en moyenne $60 \%$ ) et leur morphologie chez des hommes hypofertiles. Des changements sont advenus bien que les niveaux sanguins de ces substances n'étaient pas déficients avant le traitement. II reste toutefois à établir que l'administration de la combinaison d'acide folique et de zinc pourrait améliorer la fertilité.

\section{Huile de graines et lignans}

À part l'acide alpha linolénique (voir ci-dessus), la linette (ou graine de lin) contient plusieurs lignans, qui sont transformés en entérodiol et entérolactone dans les intestins. Ce sont des phyto-œstrogènes à effet œstrogène très faible, mais l'entérolactone inhibe l'aromatase, réduisant la conversion des androgènes (androsténédione et testostérone) en œstrogènes actifs (œstrone et œstradiol- 17 beta) $[1 ; 77]$. Par conséquent, les compléments alimentaires à base d'huile de lin ou de lignans diminuent la charge d'œstrogènes de l'organisme. On a observé que des hommes combinant une oligozoospermie avec des concentrations de FSH et d'Inhibine B normales dans le sérum présentent couramment un hyper-oestrogénisme relatif, éventuellement lié à une surcharge pondérale [45], qui peut être corrigé par un apport en lignans.

\section{Extraits de plantes}

Appliquant des techniques immuno-histochimiques, Mayerhofer et al. [46] ont prouvé récemment que la cyclo-oxygénase iso-enzyme 2 (COX-2), qui transforme l'acide arachidonique (20:4 w6) en prostaglandine inflammatoire, est présente dans le tissu interstitiel des testicules de patients atteints d'une oligozoospermie idiopathique, mais pas chez des hommes ayant une spermatogenèse normale. L'extrait d'écorce de pin maritime (Pycnogenol ${ }^{\circledast}$ ) contient des substances qui inhibent l'enzyme $\operatorname{COX}[4,54]$, réduisent la cytokine interleukine-1 bêta inflammatoire [7], et protègent les effets de la vitamine $E$ sur les cellules endothéliales [74]. Dans une étude ouverte, l'administration orale de 200 mg par jour de cet extrait a amélioré la morphologie des spermatozoïdes d'en moyenne $99 \%$ [56].

L'extrait de Lepidium Meyenii (ou extrait de Maca), une plante poussant dans les Andes Péruviennes, améliore la fonction sexuelle des individus mâles chez des souris et des rats [82], et revigore la spermatogenèse aux stades mitotiques [24]. Lorsqu'administré à des hommes ayant une spermatogenèse normale, l'extrait augmente significativement la production de spermatozoïdes $(+85 \%)$ et la motilité $(+15 \%)$ sans interférer avec la régulation endocrinienne [23].

De toute évidence, des extraits de plantes particulières produisent des effets intéressants et prometteurs pour l'avenir.

\section{Arginine et kallicréine}

Depuis plusieurs années, l'arginine $[2,17]$ et la kallicréine [60] sont recommandées pour traiter les hommes atteints d'une oligozoospermie. Les prétendus effets favorables de ces compléments alimentaires sur le traitement de patients atteints d'une oligozoospermie idiopathique ont été mis en doute dans d'autres études [11, 53].

\section{DONNEES ESSENTIELLES}

- L'infertilité masculine est le plus souvent le résultat d'influences synergistiques négatives exercées par plusieurs groupes de facteurs.

- Des facteurs externes, liés au mode de vie et à l'exposition à l'environnement, renforcent les effets des dommages testiculaires congénitaux ou acquis, par une toxicité directe, une perturbation hormonale et une surcharge d'espèces d'oxygène réactif.

- La lutte contre l'obésité, la correction d'un régime inadapté, le bannissement de l'abus de tabac et d'alcool font partie de l'approche holistique de l'infertilité masculine.

- Les nutraceutiques sont des compléments alimentaires judicieusement formulés, contenant des vitamines, des antioxydants, des minéraux et des extraits de plantes particulières.

- Il existe des preuves scientifiques que le traitement complémentaire au moyen d'un nutraceutique approprié améliore le potentiel de fertilisation des patients à fertilité amoindrie.

\section{REFERENCES}

1. ADLERCREUTZ H., BANNWART C., WAHALA K. et al. : Inhibition of human aromatase by mammalian lignans and isoflavonoid phytoestrogens. J. Steroid Biochem. Mol. Biol., 1993, 44 : 147-153.

2. AYDIN S., INCI O., ALAGOL B. : The role of arginine, indomethacin and kallikrein in the treatment of oligoasthenospermia. Int. Urol. Nephrol., 1995, 27 : 199-202.

3. BARTELLINI M., CANALE D., IZZO P.L., GIORGI P.M., MESCHINI P., MECHINI-FABRIS G.F. : L-carnitine and acetylcarnitine in human sperm with normal and reduced motility. Acta Eur. Fertil., 1987, 18: 29-31.

4. BAUMANN J., VON BRUCHHAUSEN F., WURM G. : Flavonoids and related compounds as inhibition of arachidonic acid peroxidation. Prostaglandins, 1980, $20: 627-639$.

5. BERNARD D., CHRISTOPHE A., DELANGHE J., LANGLOIS M., DE BUYZERE M., COMHAIRE F. : The effect of supplementation with an antioxidant preparation on LDL-oxidation is determined by haptoglobin polymorphism. Redox. Rep., 2003, $8: 41-46$.

6. BONDE J.P., JOFFE M., APOSTOLI P. et al. : Sperm count and chromatin structure in men exposed to inorganic lead: lowest adverse effect levels. Occup. Environ. Med., 2002, 59 : 234-242.

7. CHO K.J., YUN C.H., PACKER L., CHUNG A.S. : Inhibition mechanisms of bioflavonoids extracted from the bark of Pinus maritima on the expression of proinflammatory cytokines. Ann. N. Y. Acad. Sci., 2001, 928 : 141-156.

8. CHRISTOPHE A., ZALATA A., MAHMOUD A., COMHAIRE F. Fatty acid composition of sperm phospholipids and its nutritional implications. Middle East Fert. Soc. J., 1998, 3:46-53. 
9. CINTI D.L., COOK L., NAGI M.N., SUNEJA S.K. : The fatty acid chain elongation system of mammalian endoplasmic reticulum. Prog. Lipid Res., 1992, 31 : 1-51.

10. COMHAIRE F. : Clinical andrology: from evidence-base to ethics. The ' $E$ ' quintet in clinical andrology. Hum. Reprod., 2000, $15: 2067-2071$

11. COMHAIRE F. , VERMEULEN L. : Effect of high dose oral Kallikrein treatment in men with idiopathic subfertility: evaluation by means of in vitro penetration test of zona free hamster ova. Int. J. Androl., 1983, $6:$ 168-172.

12. COMHAIRE F.H., CHRISTOPHE A.B., ZALATA A.A., DHOOGE W.S., MAHMOUD A.M., DEPUYDT C.E. : The effects of combined conventional treatment, oral antioxidants and essential fatty acids on sperm biology in subfertile men. Prostaglandins Leukot. Essent. Fatty Acids, 2000, 63 : 159-165.

13. COMHAIRE F.H., GAREM Y.F.E., MAHMOUD A., EERTMANS F., SCHOONJANS F. : Combined conventional/antioxidant "Astaxanthin" treatment for male infertility : a double blind randomized trial. Asian J. Androl., 2004, sous presse.

14. COMHAIRE F.H. , MAHMOUD A.M. : Editorial commentary. J. Androl., 2004, $25: 771-772$.

15. COSTA M., CANALE D., FILICORI M., D'LDDIO S., LENZI A. : L-carnitine in idiopathic asthenozoospermia : a multicenter study. Italian Study Group on Carnitine and Male Infertility. Andrologia, 1994, $26:$ 155-159.

16. DAWSON E.B., HARRIS W.A., TETER M.C., POWELL L.C. : Effect of ascorbic acid supplementation on the sperm quality of smokers. Fertil. Steril,, 1992, $58: 1034-1039$.

17. DE ALOYSIO D., MANTUANO R., MAULONI M., NICOLETTI G. : The clinical use of arginine aspartate in male infertility. Acta Eur. Fertil., 1982, $13: 133-167$

18. DEBAUN M.R., NIEMITZ E.L., FEINBERG A.P. : Association of in vitro fertilization with Beckwith-Wiedemann syndrome and epigenetic alterations of LIT1 and H19. Am. J. Hum. Genet., 2003, 72 : 156-160.

19. DEPUYDT C.E., MAHMOUD A.M., DHOOGE W.S., SCHOONJANS F.A., COMHAIRE F.H. : Hormonal regulation of inhibin $B$ secretion by immature rat sertoli cells in vitro : possible use as a bioassay for estrogen detection. J. Androl., 1999, $20: 54-62$.

20. EDWARDS R.G. , LUDWIG M. : Are major defects in children conceived in vitro due to innate problems in patients or to induced genetic damage ? Reprod. Biomed. Online, 2003, 7 : 131-138.

21. FRAGA C.G., MOTCHNIK P.A., SHIGENAGA M.K., HELBOCK H.J., JACOB R.A., AMES B.N. : Ascorbic acid protects against endogenous oxidative DNA damage in human sperm. Proc. Natl. Acad. Sci. U. S. A., 1991, 88 : 11003-11006.

22. GOLAN R., WEISSENBERG R., LEWIN L.M. : Carnitine and acetylcarnitine in motile and immotile human spermatozoa. Int. J. Androl., 1984, $7:$ 484-494.

23. GONZALES G.F., CORDOVA A., GONZALES C., CHUNG A., VEGA K., VILLENA A. : Lepidium meyenii (Maca) improved semen parameters in adult men. Asian J. Androl., 2001, 3 : 301-303.

24. GONZALES G.F., RUIZ A., GONZALES C., VILLEGAS L., CORDOVA A. : Effect of Lepidium meyenii (maca) roots on spermatogenesis of male rats. Asian J. Androl., 2001, $3: 231$ 233.

25. GOTO S., KOGURE K., ABE K. et al. : Efficient radical trapping at the surface and inside the phospholipid membrane is responsible for highly potent antiperoxidative activity of the carotenoid astaxanthin. Biochim. Biophys. Acta, 2001, 1512 : 251-258.

26. GREEN N.S. : Risks of birth defects and other adverse outcomes associated with assisted reproductive technology. Pediatrics, 2004, $114: 256-259$

27. HANSEN M., KURINCZUK J.J., BOWER C., WEBB S. : The risk of major birth defects after intracytoplasmic sperm injection and in vitro fertilization. N. Engl. J. Med., 2002, 346 : 725730.

28. HUGHES C.M., LEWIS S.E., MCKELVEY-MARTIN V.J., THOMPSON W. : The effects of antioxidant supplementation during Percoll preparation on human sperm DNA integrity. Hum. Reprod., 1998, $13: 1240-1247$.

29. HURTADO DE CATALFO G.E., DE GOMEZ DUMM I.N. : Polyunsaturated fatty acid biosynthesis from [1-14C]20:3 n-6 acid in rat cultured Sertoli cells. Linoleic acid effect. Int. J. Biochem. Cell Biol., 2002, 34 : 525-532.

30. IRVINE D.S., TWIGG J.P., GORDON E.L., FULTON N., MILNE P.A., AITKEN R.J. : DNA integrity in human spermatozoa : relationships with semen quality. J. Androl., 2000, 21 : $33-44$

31. IWAMOTO T., HOSODA K., HIRANO R. et al. : Inhibition of low-density lipoprotein oxidation by astaxanthin. J. Atheroscler. Thromb., 2000, $7: 216-222$.

32. JEULIN C. , LEWIN L.M. Role of free L-carnitine and acetyl-Lcarnitine in post-gonadal maturation of mammalian spermatozoa. Hum. Reprod. Update, 1996, $2:$ 87-102.

33. KATZ P., NACHTIGALL R., SHOWSTACK J. : The economic impact of the assisted reproductive technologies. Nat. Cell Biol., 2002, 4 Suppl : s29-s32.

34. KENT-FIRST M.G., KOL S., MUALLEM A. et al. : The incidence and possible relevance of Y-linked microdeletions in babies born after intracytoplasmic sperm injection and their infertile fathers. Mol. Hum. Reprod., 1996, 2 : 943-950.

35. KESSOPOULOU E., POWERS H.J., SHARMA K.K. et al. : A double-blind randomized placebo cross-over controlled trial using the antioxidant vitamin $E$ to treat reactive oxygen species associated male infertility. Fertil. Steril., 1995, 64 : 825-831.

36. KOHENGKUL S., TANPHAICHITR V., MUANGMUN V., TANPHAICHITR N. : Levels of L-carnitine and L-O-acetylcarnitine in normal and infertile human semen: a lower level of L-O-acetycarnitine in infertile semen. Fertil. Steril., 1977, $28: 1333-$ 1336.

37. KOUDSTAAL J., BRUINSE H.W., HELMERHORST F.M., VERMEIDEN J.P., WILLEMSEN W.N., VISSER G.H. Obstetric outcome of twin pregnancies after in vitro fertilization : a matched control study in four Dutch University hospitals. Hum. Reprod., 2000, $15: 935-940$.

38. LAMBERT R.D. Safety issues in assisted reproduction technology : the children of assisted reproduction confront the responsible conduct of assisted reproductive technologies. Hum. Reprod., 2002, 17 : 3011-3015.

39. LENZI A., CULASSO F., GANDINI L., LOMBARDO F., DONDERO F. : Placebo-controlled, double-blind, cross-over trial of glutathione therapy in male infertility. Hum. Reprod., 1993, 8 : 1657-1662.

40. LENZI A., LOMBARDO F., SGRO P. et al. : Use of carnitine therapy in selected cases of male factor infertility : a doubleblind crossover trial. Fertil. Steril., 2003, 79 : 292-300.

41. LEWIN A. , LAVON $H$. : The effect of coenzyme $Q 10$ on sperm motility and function. Mol. Aspects Med., 1997, 18 Suppl : S213-9. 
42. MA K., SHARKEY A., KIRSCH S. et al. : Towards the molecular localisation of the AZF locus : mapping of microdeletions in azoospermic men within 14 subintervals of interval 6 of the human Y chromosome. Hum. Mol. Genet., 1992, 1 : 29-33.

43. MAHER E.R., AFNAN M., BARRATT C.L. : Epigenetic risks related to assisted reproductive technologies: epigenetics, imprinting, ART and icebergs ? Hum. Reprod., 2003, 18 : 2508-2511.

44. MAHMOUD A., KISS P., KAUFMAN J.M., COMHAIRE F., ASCLEPIOS. : The influence of age and lead exposure on inhibin B serum levels in men. Int. J. Androl., 2000, 23 (suppl. 1) : P094.

45. MAHMOUD A.M., COMHAIRE F.H., DEPUYDT C.E. : The clinical and biological significance of serum inhibins in subfertile men. Reprod. Toxicol., 1998, 12 : 591-599.

46. MAYERHOFER A., MEINEKE V., KÖHN F.M., FRUNGIERI M. Cyclooxygenase (COX-2) in male infertility: a new link between prostaglandins and testicular fibrosis? Andrologia, 2002, $34: 272-273$

47. MEACHEM S.J., NIESCHLAG E., SIMONI M. : Inhibin B in male reproduction : pathophysiology and clinical relevance. Eur. J. Endocrinol., 2001, $145:$ 561-571.

48. MENCHINI-FABRIS G.F., CANALE D., IZZO P.L., OLIVIERI L., BARTELLONI M. : Free L-carnitine in human semen: its variability in different andrologic pathologies. Fertil. Steril., 1984, 42: 263-267

49. MOLL A.C., IMHOF S.M., CRUYSBERG J.R., SCHOUTENVAN MEETEREN A.Y., BOERS M., VAN LEEUWEN F.E. : Incidence of retinoblastoma in children born after in vitro fertilisation. Lancet, 2003, $361: 309-310$.

50. MONCADA M.L., VICARI E., CIMINO C., CALOGERO A.E., MONGIOI A., D'AGATA R. : Effect of acetylcarnitine treatment in oligoasthenospermic patients. Acta Eur. Fertil., 1992, 23 : 221-224.

51. PALERMO G., JORIS H., DEVROEY P., VAN STEIRTEGHEM A.C. : Pregnancies after intracytoplasmic injection of single spermatozoon into an oocyte. Lancet, 1992, 340:17-18.

52. PINBORG A., LOFT A., SCHMIDT L., GREISEN G., RASMUSSEN S., ANDERSEN A.N. : Neurological sequelae in twins born after assisted conception : controlled national cohort study. Br. Med. J., 2004, $329: 311$

53. PRYOR J.P., BLANDY J.P., EVANS P., CHAPUT D.S.D., USHERWOOD M. : Controlled clinical trial of arginine for infertile men with oligozoospermia. Br. J. Urol., 1978, $50: 47-50$.

54. ROHDEWALD P. : A review of the French maritime pine bark extract (Pycnogenol), a herbal medication with a diverse clinical pharmacology. Int. J. Clin. Pharmacol. Ther., 2002, 40 : 158-168.

55. ROLF C., COOPER T.G., YEUNG C.H., NIESCHLAG E. : Antioxidant treatment of patients with asthenozoospermia or moderate oligoasthenozoospermia with high-dose vitamin C and vitamin $E$ : a randomized, placebo-controlled, doubleblind study. Hum. Reprod., 1999, 14 : 1028-1033.

56. ROSEFF S.J. , GULATI R. : Improvement of sperm quality by Pycnogenol. Eur. Bull. Drug Res., 1999, 7 : 33-36.

57. RUIZ-PESINI E., ALVAREZ E., ENRIQUEZ J.A., LOPEZPEREZ M.J. : Association between seminal plasma carnitine and sperm mitochondrial enzymatic activities. Int. J. Androl., 2001, $24: 335-340$.

58. SAETHER T., TRAN T.N., ROOTWELT H., CHRISTOPHERSEN B.O., HAUGEN T.B. : Expression and regulation of delta5-desaturase, delta6-desaturase, stearoyl-coenzyme $A$
(CoA) desaturase 1, and stearoyl-CoA desaturase 2 in rat testis. Biol. Reprod., 2003, 69 : 117-124.

59. SCHIEVE L.A., RASMUSSEN S.A., BUCK G.M., SCHENDEL D.E., REYNOLDS M.A., WRIGHT V.C. : Are children born after assisted reproductive technology at increased risk for adverse health outcomes ? Obstet. Gynecol., 2004, 103 : 1154-1163.

60. SCHILL W.B., KRIZIC A., RJOSK H. : Determination of various semen parameters and sex hormone levels in subfertile men during kallikrein therapy. Adv. Exp. Med. Biol., 1979, 120A: 537-546.

61. SCOTT R., MACPHERSON A., YATES R.W., HUSSAIN B., DIXON J. : The effect of oral selenium supplementation on human sperm motility. Br. J. Urol., 1998, 82 : 76-80.

62. SHARPE R.M. The 'oestrogen hypothesis'- where do we stand now ? Int. J. Androl., 2003, 26: 2-15

63. SKAKKEBAEK N.E. : Testicular dysgenesis syndrome : new epidemiological evidence. Int. J. Androl., 2004, 27 : 189-191.

64. SOUFIR J.C., DUCOT B., MARSON J. et al. : Levels of seminal free $L(-)$ carnitine in fertile and infertile men. Int. J. Androl., 1984, $7: 188-197$.

65. STEPTOE P.C., EDWARDS R.G. : Birth after the reimplantation of a human embryo. Lancet, 1978, $2: 366$.

66. STROMBERG B., DAHLQUIST G., ERICSON A., FINNSTROM O., KOSTER M., STJERNQVIST K. : Neurological sequelae in children born after in vitro fertilisation : a population-based study. Lancet, 2002, $359: 461-465$.

67. SUTCLIFFE A.G., TAYLOR B., LI J., THORNTON S., GRUDZINSKAS J.G., LIEBERMAN B.A. : Children born after intracytoplasmic sperm injection : population control study. Br. Med. J., 1999, 318: 704-705.

68. TANPHAICHITR N. : in vitro stimulation of human sperm motility by acetylcarnitine. Int. J. Fertil., 1977, $22: 85-91$.

69. TIEPOLO L., ZUFFARDI O. : Localization of factors controlling spermatogenesis in the nonfluorescent portion of the human $Y$ chromosome long arm. Hum. Genet., 1976, 34 : 119-124.

70. TRAN T.N., RETTERSTOL K., CHRISTOPHERSEN B.O. : Metabolism of long-chain polyunsaturated fatty acids in testicular cells. In : De Vriese S.R., Christophe A.B. eds. Male fertility and lipid metabolism. Illinois, AOCS Press, 2003 : 11-22.

71. VAN DER VEN K., PESCHKA B., MONTAG M. et al. : Increased frequency of congenital chromosomal aberrations in female partners of couples undergoing intracytoplasmic sperm injection. Hum. Reprod., 1998, $13: 48-54$.

72. VAN DISSEL-EMILIANI F.M., GROOTENHUIS A.J., DE JONG F.H., DE ROOIJ D.G. : Inhibin reduces spermatogonial numbers in testes of adult mice and Chinese hamsters. Endocrinology, 1989, $125: 1899-1903$.

73. VANHOORNE M., COMHAIRE F., DE BACQUER D. : Epidemiological study of the effects of carbon disulfide on male sexuality and reproduction. Arch. Environ. Health, 1994, 49 : 273-278.

74. VIRGILI F., KIM D., PACKER L. : Procyanidins extracted from pine bark protect alpha-tocopherol in ECV 304 endothelial cells challenged by activated RAW 264.7 macrophages: role of nitric oxide and peroxynitrite. FEBS Lett., 1998, 431 : 315318.

75. VITALI G., PARENTE R., MELOTTI C. : Carnitine supplementation in human idiopathic asthenospermia : clinical results. Drugs Exp. Clin. Res., 1995, 21 : 157-159.

76. VOLIANI S., BERTOZZI M.A., ROSSI P., MENCHINI-FABRIS 
G.F. : The treatment of male infertility with L-carnitine/L-acetyl carnitine. XVII National Congress, Societa Italiana Andrologia SIA, Venezia 2001, 2001, $8: 122$.

77. WANG C., MAKELA T., HASE T., ADLERCREUTZ H., KURZER M.S. : Lignans and flavonoids inhibit aromatase enzyme in human preadipocytes. J. Steroid Biochem. Mol. Biol., 1994, $50: 205-212$.

78. WENNERHOLM U., BERGH C., HAMBERGER L. et al. : Incidence of congenital malformations in children born after ICSI. Hum. Reprod., 2000, 15 : 944-948.

79. WILDMAN R.E.C., MEDEIROS D.M. : Advanced human nutrition. London, CRC Press, $2000: 370-371$.

80. WONG W.Y., MERKUS H.M., THOMAS C.M., MENKVELD R., ZIELHUIS G.A., STEEGERS-THEUNISSEN R.P. : Effects of folic acid and zinc sulfate on male factor subfertility : a doubleblind, randomized, placebo-controlled trial. Fertil. Steril., 2002, $77: 491-498$.

81. ZALATA A.A., CHRISTOPHE A.B., DEPUYDT C.E., SCHOONJANS F., COMHAIRE F.H. : The fatty acid composition of phospholipids of spermatozoa from infertile patients. Mol. Hum. Reprod., 1998, 4 : 111-118.

82. ZHENG B.L., HE K., KIM C.H. et al. : Effect of a lipidic extract from lepidium meyenii on sexual behavior in mice and rats. Urology, 2000, $55:$ 598-602.

Communication au $X X 1^{\circ}$ Congrès de la Société d'Andrologie de Langue Française, Clermont-Ferrand, 9-11 Décembre 2004.

Manuscrit reçu : décembre 2004 ; accepté décembre 2004

\begin{abstract}
Nutraceuticals and food supplementation for the treatment of the subfertile male

Frank COMHAIRE, Ahmed MAHMOUD

Several controlled and well-validated trials provide evidence that food supplementation with particular substances can improve semen quality and function of subfertile men. These include the antioxidants astaxanthin, tocopherols and ubiquinone Q10, essential fatty acids of the omega-3 group, zinc, and folic acid. There is suggestive evidence that certain plant extracts and lignans may equally exert beneficial effects. Supplementation with a nutraceutical (Qualisperm ${ }^{\circledR}$, Nutriphyt, Oostkamp, Belgium) containing these substances increases the probability of spontaneous conception and successful pregnancy. Although the exact mechanisms of action of these supplements on spermatogenesis and sperm function remain to be unravelled, a direct effect on the cells of Sertoli and via epididymal function seems conceivable. Nutraceutical food supplementation should also be considered before in vitro fertilization and ICSI, in order to reduce the oxidative damage to sperm DNA.
\end{abstract}

Key words : : nutraceuticals, infertility, fatty acids, antioxidants, astaxanthin 